\title{
Nonlinear standing waves on planar branched systems: shrinking into metric graph
}

\author{
Z. Sobirov ${ }^{1}$, D. Babajanov ${ }^{2}$ and D. Matrasulov ${ }^{2}$ \\ ${ }^{1}$ Tashkent Financial Institute, 60A, Amir Temur Str., 100000, Tashkent, Uzbekistan \\ ${ }^{2}$ Turin Polytechnic University in Tashkent, 17 Niyazov Str., 100095, Tashkent, Uzbekistan \\ sobirovzar@gmail.com
}

PACS 05.45.Yv, 42.65.Wi, 42.65.Tg

DOI 10.17586/2220-8054-2017-8-1-29-37

\begin{abstract}
We treat the stationary nonlinear Schrödinger equation on two-dimensional branched domains, so-called fat graphs. The shrinking limit when the domain becomes one-dimensional metric graph is studied by using analytical estimate of the convergence of fat graph boundary conditions into those for metric graph. Detailed analysis of such convergence on the basis of numerical solution of stationary nonlinear Schrödinger equation on a fat graph is provided. The possibility for reproducing different metric graph boundary conditions studied in earlier works is shown. Practical applications of the proposed model for such problems as Bose-Einstein condensation in networks, branched optical media, DNA, conducting polymers and wave dynamics in branched capillary networks are discussed.
\end{abstract}

Keywords: metric graph, Schrödinger equation.

Received: 11 August 2016

Revised: 4 September 2016

\section{Introduction}

Branched structures and networks appear in many physical systems and in complex systems from biology, ecology, sociology, economy and finance [1,2]. Particle and wave dynamics in such systems can be effectively modeled by nonlinear evolution equations on metric graphs. The latter are one dimensional system of bonds which are connected at one or more vertices (branching points). The connection rule is called the topology of the graph. When the bonds can be assigned a length, the graph is called a metric graph. Recently nonlinear evolution equations on metric graphs have attracted much attention in the literature (see the Refs. [3-10]). To solve nonlinear evolution equations on networks, one needs to impose boundary conditions on the graph vertices. Soliton solutions providing reflectionless transmission at the graph vertex together with integrable boundary conditions were derived in [3]. Different aspects of the nonlinear Schrödinger equation, including soliton solutions, are discussed in the Refs. [5,6]. Solutions of the stationary nonlinear Schrödinger equation on graphs for different vertex conditions were obtained in [4,5,8-10]. Despite the growing interest in nonlinear evolution equations and soliton dynamics in networks, most studies are still restricted by considering metric graphs, i.e. by one-dimensional motion wave motion in branched structures. However, in many cases, particle and wave motion in branched structures have certain transverse components, so that the system is two-dimensional. Such systems should be described using two-dimensional evolution equations on planar networks. Such systems can be modeled by so-called fat graphs. Previously, the linear Schrödinger equation on fat graphs was addressed in a number of works [11-13] by considering metric graph limit as transition to from planar to linear wave motions. Extension of such a study to the case of nonlinear Schrödinger equation based on the numerical treatment of the problem was done in recent work [14]. Fat graph treatment of the sine-Gordon equation for branched Y-junctions was numerically treated in [15]. Pioneering treatment of nonlinear evolution equations on fat graphs date back to Kosugi, who presented in Ref. [16] a strict mathematical treatment of the nonlinear elliptic differential equations on branched domains and estimates for the shrinking limit. The study of the nonlinear differential equation on branched domains is complicated due to the nonlinearity of the boundary conditions imposed at the branching area or vertex. In the case of nonlinear evolution equations related to physics, such boundary conditions should be derived from conservation laws or other physical conditions that greatly simplifies these boundary conditions $[3,14]$.

In this work, we address the problem of the stationary (time-independent) nonlinear Schrödinger equation on fat graphs by focusing on metric graph limit in the shrinking of a fat graph. In particular, we obtain an estimate for the vertex boundary conditions in the shrinking limit and show that they reproduce those for the metric graph considered in $[5,8]$. Also, we present a treatment of the problem on the basis of numerical solution of stationary NLSE on fat graph. For small enough bond widths the numerical solution reproduces the metric graph solutions from Refs. [5,8]. 
Motivation for the study of the stationary NLSE comes from such problems as Bose-Einstein condensation on networks [17-22], standing waves in branched optical waveguides [23], branched liquid crystals and standing wave-polarons in polymers $[24,25]$. All these systems require taking into account of transverse component of the wave motion which can be done by considering stationary NLSE on fat graphs. Estimating of the "boundary" between the planar and linear motions is of importance for experiments on the study of wave motion in nonlinear networks, as such networks are always somehow planar or tubular.

This paper is organized as follows. In the next section, we give formulation of stationary NLSE on fat and metric graphs. Section III presents the results for numerical solution of the stationary NLSE on fat graph at different values of the graphs width by considering shrinking limit. In section IV, we present detailed discussion of potential application of fat graph problem to some physical systems having planar branched structure. Finally, section $\mathrm{V}$ gives some concluding remarks.

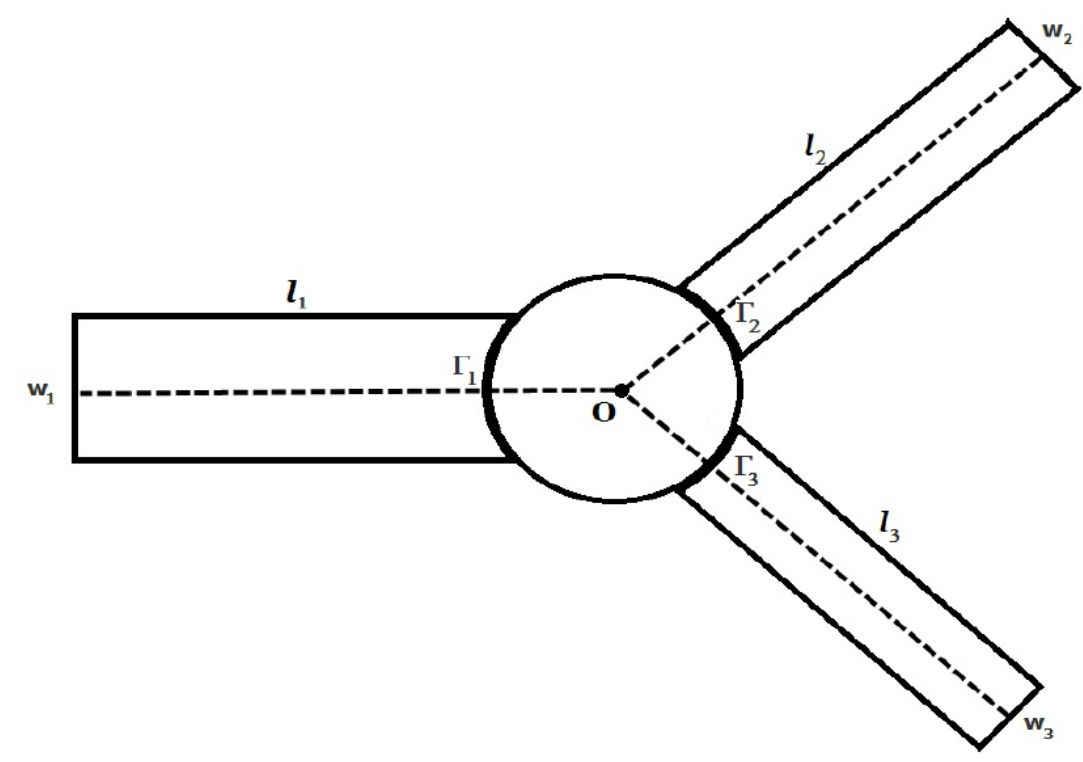

FIG. 1. Sketch of star-shaped fat and metric graphs. Dashed line presents metric graph

\section{Stationary NLSE on a fat graph}

Wave equations on planar branched domains, which are often called "fat graphs", have attracted much attention in the context of wave dynamics during last decade. In particular, stationary linear wave equations on fat graphs have been studied in the Refs. [11-13,26-28] (see [29,30] and references therein for detailed reviews). The corresponding nonlinear problem has been mainly studied for the one-dimensional case by considering the metric graph approach Different aspects of the nonlinear Schrödinger equation for branched one dimensional branched domains called metric graphs were previously studied earlier [4-10]. A metric graph is a set of bonds connected to each other at the vertices according to a rule which is called the topology of a graph with metrics defined in each bond. Recently, the problem of soliton transport in planar branched domains was studied based on numerical solution of NLSE on fat graph [17]. Transition from fat to metric graph problem was shown in this study.

Our purpose is to explore solutions of the stationary nonlinear Schrödinger equation over a two-dimensional branched domain in the limit when the domain shrinks to a metric graph. Therefore, we introduce two problems: "fat graph" and the "metric graph" problems. Both fat and metric graphs are presented in Fig. 1. A fat graph is a branched domain having two dimensional bonds and vertex as presented (See Fig. 1). In the following, we denote bond-lengths of such graph by $l_{1}, l_{2}$ and $l_{3}$, bond-widths by $w_{1}, w_{2}$ and $w_{3}$. The diameter of the vertex region $\Omega_{\epsilon}=\epsilon \Omega_{1}$ is $r \epsilon$. The stationary nonlinear Schrödinger equation (NLSE) on a "fat graph" is given as:

$$
-\Delta \psi+\left(V_{\alpha, \epsilon}-\mu\right) \psi-|\psi|^{2} \psi=0,
$$

where the potential:

$$
V_{\alpha, \epsilon}(x)=-\frac{\alpha}{2 \pi \epsilon} \exp \left(-|x|^{2} / 2 \epsilon^{2}\right)
$$


is localized at the vertex domain, $\alpha \in R$ is a parameter. The potential is chosen as to reproduce delta-function in the shrinking limit, i.e.:

$$
V_{\alpha, \epsilon} \sim-\alpha \epsilon \delta(x) \text { for } \epsilon \rightarrow 0 \text {. }
$$
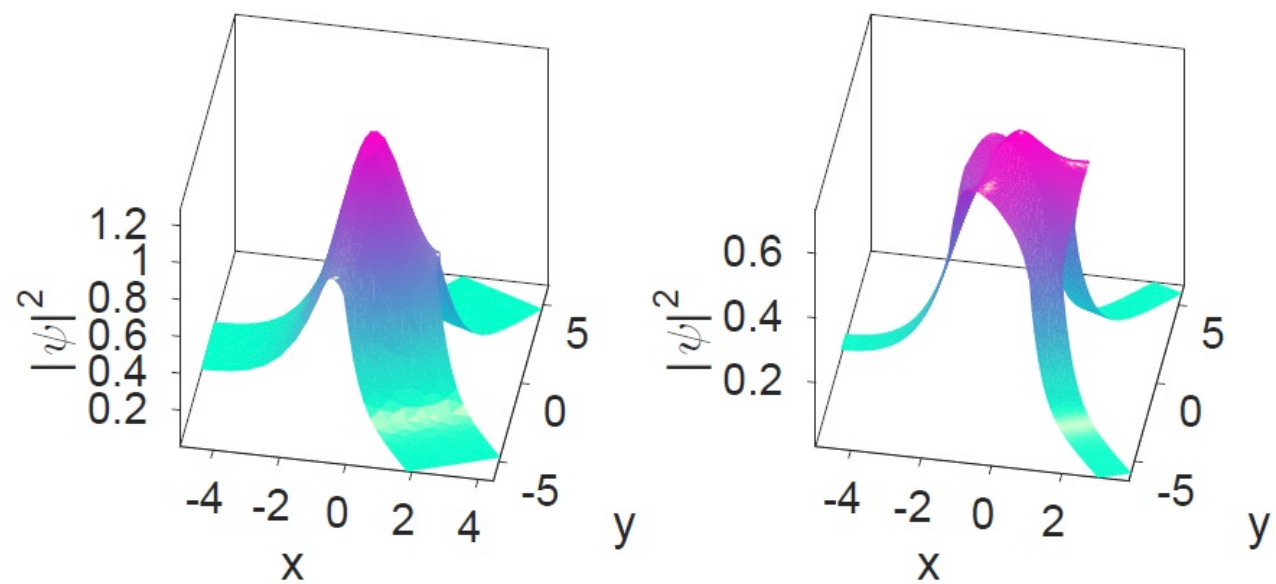

FIG. 2. Solution of NLSE on a fat graph at $\epsilon=3$ and $1, \mu=-1, \alpha=0.5$

In the metric graph case, we determine the coordinate for the $k$ th bond from 0 to $l_{k}, k=1,2,3$. The metric graph problem is determined by the stationary NLSE which is given for each bond of the graph as:

$$
-\phi^{\prime \prime}-\mu \phi-|\phi|^{2} \phi=0 \text {. }
$$

These equations are related via the vertex conditions given by:

$$
\begin{gathered}
\phi_{1}=\phi_{2}=\phi_{3}, \\
\phi_{1}^{\prime}+\phi_{2}^{\prime}+\phi_{3}^{\prime}=\alpha \phi_{1} .
\end{gathered}
$$

In the following, the problems given by Eqs.(4)-(6) will be called "metric graph problem". The aim of this paper is to explore both analytically and numerically the shrinking limit of the fat graph problem given by Eqs. (1) and (2) and determining the conditions providing in the shrinking limit the transition of the fat graph problem to that of the metric graph. Such an analysis will consist of two parts: 1) an analytical estimate for the convergence of the fat graph problem to that of the metric graph and 2) numerical analysis for such convergence. The latter implies the analysis of numerical solution solution of NLSE for fat graph for different small values of the bond and vertex widths. Here, we focus on the vertex conditions considered in Refs. [5, 7,9] which are often called "delta" type boundary conditions.

We divided the fat graph into the following parts: the first is the vertex region denoted by $\Omega_{\epsilon}=\epsilon \Omega_{1}$, where we assumed $\Omega_{1}$ is a convex region with smooth enough boundary $\left(0 \in \Omega_{1}\right)$ and tabular (rectangular) parts. In the limit $\epsilon \rightarrow 0$, the vertex region is given as a vertex point 0 , while tabular parts tends to the bonds of metric graph. The convergence problem in the case of the tabular region is well studied. Here, we refer to [16,31]. We only focus on the vertex region and denote by $\Gamma_{k, \epsilon},(k=1,2,3)$ the those parts(arcs) of the vertex which are connected to the bonds $\left(\Gamma_{k, \epsilon}=\epsilon \Gamma_{k}\right.$ ). We impose the following boundary conditions for NLSE given by Eq. (1) in $\Omega_{\epsilon}$ :

$$
\frac{\partial \psi}{\partial n}=0 \text { on } \partial \Omega_{\varepsilon} /\left(\Gamma_{1, \varepsilon} \cup \Gamma_{2, \varepsilon} \cup \Gamma_{3, \varepsilon}\right) .
$$

We assume that:

$$
\left.\frac{\partial \psi}{\partial n}\right|_{\Gamma_{k, \varepsilon}} \rightarrow \varphi_{k}^{\prime},\left.\quad \psi\right|_{\Gamma_{k, \varepsilon}} \rightarrow \varphi_{k} \text { at } \epsilon \rightarrow 0, k=1,2,3
$$

where $\varphi_{k}$ and $\varphi_{k}^{\prime}(k=1,2,3)$ are constants.

First, we show that $\varphi_{1}=\varphi_{2}=\varphi_{3}$.

According to [16,31] and maximum principle [32], we have $|\psi(x)|<c_{1},|\nabla \psi(x)| \leq c_{2}$ for $x \in \Omega_{\epsilon}$

We denote $v(y)=\psi(y / \epsilon)$. The function $v(y)$ satisfies the following equation:

$$
-\Delta_{y} v(y)-\epsilon^{2} f(v(y))+\alpha \epsilon V_{1,1} v(y)=0,
$$

with $f(v)=|v|^{2} v+\mu v$. 


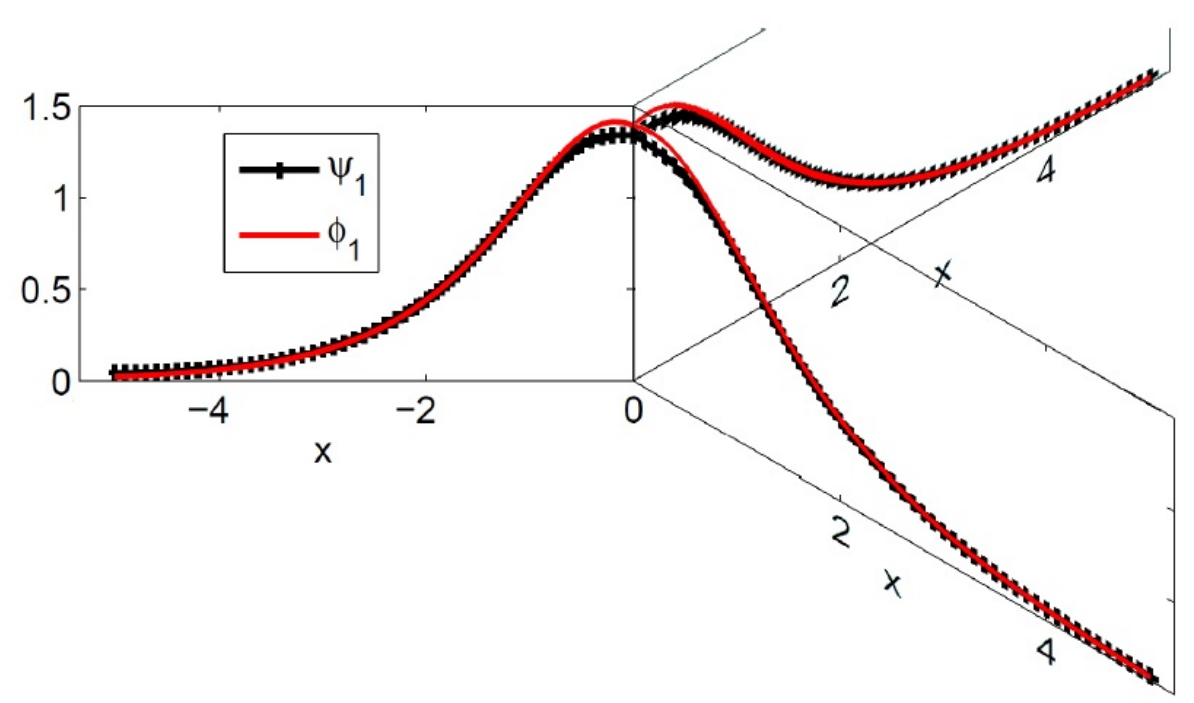

FIG. 3. Solution of NLSE on a fat graph at at $\epsilon=0.5, \mu=-1, \alpha=0.5$

The function $v(y)$ satisfies the estimate (see, e.g. $[16,32])\|v\|_{C^{2}\left(\Omega_{1}\right)} \leq C_{3}$. From the Ascoli-Arzel theorem, there exist a sequence $\epsilon_{m} \rightarrow 0$ and the function $v_{\infty} \in C^{1}\left(\overline{\Omega_{1}}\right)$, such that $\lim _{k \rightarrow \infty}\left\|v_{m}-v_{\infty}\right\|_{C^{1}\left(\overline{\Omega_{1}}\right)}=0$. Where $v_{m}(y)$ is the function $v(y)$ with $\epsilon=\epsilon_{m}$.

Then we have

$$
\begin{aligned}
\iint_{\Omega_{1}}\left|\nabla_{y} v_{m}(y)\right|^{2} d y & =\int_{\partial \Omega_{\epsilon_{m}}} \psi_{m} \frac{\partial \psi_{m}}{\partial n} d s_{x}-f-\iint \Omega_{\epsilon_{m}}\left[V_{\alpha, \epsilon_{m}}(x) \psi_{m}(x)+u_{m}(x) f\left(\psi_{m}(x)\right)\right] d x \\
& \leq c_{1} c_{2} \epsilon_{m} \sum_{k=1}^{3}\left|\Gamma_{k}\right|+c_{1}^{4}\left|\Omega_{1}\right| \epsilon_{m}^{2}+c_{1} \epsilon_{m} \rightarrow 0 .
\end{aligned}
$$

From Eq. (9) we have $v_{\infty}=$ const, which proves $\varphi_{1}=\varphi_{2}=\varphi_{3}=\varphi$.

Next, we verify the second vertex condition in the limiting problem. For mean values of the normal derivatives on the boundary we have $\left(\epsilon=\epsilon_{m}\right)$ :

$$
\sum_{k=1}^{3} \frac{1}{\epsilon} \int_{\Gamma_{k, \epsilon}} \frac{\partial \psi}{\partial n} d \Gamma_{k, \epsilon} \rightarrow \sum_{k}\left|\Gamma_{1}\right| \varphi_{k}^{\prime}
$$

Conversely, integrating Eq. (10) over $\Omega_{\epsilon}$, we get:

$$
\sum_{k=1}^{3} \frac{1}{\epsilon} \int_{\Gamma_{k, \epsilon}} \frac{\partial \psi}{\partial n} d \Gamma_{k, \epsilon}+\alpha \iint_{\Omega_{\epsilon}} \frac{1}{\epsilon^{2}} V_{1,1}\left(\frac{x}{\epsilon}\right)-\frac{1}{\epsilon} \iint_{\Omega_{\epsilon}}|\psi|^{2} \psi d x=\frac{\mu}{\epsilon} \iint_{\Omega_{\epsilon}} \psi d x .
$$

According to maximum principle [32], for small $\epsilon$, we have $\sup |u| \leq C$. Therefore, for the integrals we can write:

$$
\frac{1}{\epsilon} \iint_{\Omega_{\epsilon}}|\psi|^{2} \psi d x \sim O(\epsilon), \frac{\mu}{\epsilon} \iint_{\Omega_{\epsilon}} \psi d x \sim O(\epsilon)
$$

for small $\epsilon$. Taking into account the above relations and properties of the potential $V_{\alpha, \epsilon}$, we obtain:

$$
\lim _{\epsilon \rightarrow 0} \frac{1}{\epsilon} \int_{\Gamma_{k, \epsilon}} \frac{\partial \psi}{\partial n} d \Gamma_{j, \epsilon}=\alpha \psi(0)
$$

Eqs. (10) and (12) lead to

$$
\sum_{k}\left|\Gamma_{k}\right| \varphi_{k}^{\prime}=\alpha \varphi
$$


Thus, we showed convergence of the fat graph problem given by Eqs. (1), (2) and (10) to that for metric graph with delta type boundary conditions given by Eqs. (4)-(6). In the next section, we will show such convergence on the basis of the numerical solution of stationary NLSE on fat graph.

\section{Numerical treatment of the shrinking limit}

Our purpose is showing convergence of the stationary NLSE on fat graph into that for metric graph in the shrinking limit using both analytically and numerical analysis of the shrinking limit. Such convergence is of practical importance for various problems dealing with wave dynamics in branched structures where one needs to neglect by transverse motion of the waves. Here, we will focus on the analysis of wave function behavior itself as well as $|\psi(x, y)|^{2}$. The latter has important physical meaning in the practical applications of the NLSE, e.g., density of particles in BEC, beam intensity in optics, etc.

The estimate presented by Eqs. (12) and (13) shows the convergence of the vertex boundary conditions of a fat graph with those of the metric graph. Such a convergence can be shown on the basis of numerical solution for the stationary NLSE on fat graphs by considering the shrinking limit, $\epsilon \rightarrow 0$.

Here, we will explore numerical solutions of Eq. (1) with the fat graph boundary conditions given by Eq. (10) at different fat graph bond length values of the by considering both attractive and repulsive nonlinearities. These describe bright and dark (static) solitons, respectively. Assuming $w_{1}=\epsilon w_{2}=\epsilon w_{3}$, we show that these numerical solutions reproduce the solutions of metric graph problem analytically obtained in the Refs. [5]. Solution of the metric graph problem given by Eqs. (4)-(6) was obtained in [5] and can be written as:

$$
\phi_{j}(x, a)=\sqrt{2 \mu} \operatorname{sech}\left(\sqrt{\mu}\left(x-a^{j}\right)\right),
$$

where

$$
a^{j}=\frac{1}{\mu} \operatorname{artanh}\left(\frac{\alpha}{(2 j-3) \sqrt{\mu}}\right), \quad j=1,2,3 .
$$

In Fig. 2, solutions of the stationary NLSE on fat graph obtained by numerical solutions of two-dimensional stationary NLSE (1) with the boundary conditions (10) are for different values of the bond width $\epsilon=3$, and 1 $(\mu=-1, \alpha=0.5)$. As it can be seen, the wave function is localized at the vertex in all cases.

Figure 3 compares solution of Eq. (1) for the bond width, $\epsilon=1$ with that of for metric graph given by Eqs. (4)-(6). It can be observed the convergence of the solution of fat graph problem to that of metric graph in the shrinking limit.

In addition to the NLSE with attractive (focusing) nonlinearity given by Eq. (1), one can consider repulsive case when the wave equation is given as:

$$
-\Delta \psi+\left(V_{\alpha, \epsilon}-\mu\right) \psi+|\psi|^{2} \psi=0 .
$$

In Fig. 4, solutions for the fat and metric graphs are compared with the case of repulsive nonlinearity and $\epsilon=0.3$. Unlike the attractive case, the wave function is localized on the bonds for this repulsive nonlinearity. Fig. 5 shows how the solution NLSE on fat graph depends on the chemical potential $\mu$.

\section{Standing nonlinear waves in branched planar structures}

Two-dimensional branched structures, where the wave dynamics are described by the nonlinear Schrödinger equation, appear in many areas of physics. Here, we will briefly discuss physical systems where the above model can be applied.

$B E C$ in planar networks. Bose-Einstein condensation is a remarkable phenomenon that can be described by a version of NLSE which is called Gross-Pitaevskii equation [17-21]. It can be realized in trapped cold atoms and depending on the type of a trap NLSE based model has different potentials and boundary conditions. The above model describes BEC in planar branched structures/traps which can be experimentally created in several physical systems. We note that the Bose-Einstein condensation in networks attracted much attention recently (see, e.g. Refs. [17-22]). Despite the fact that the nonlinear Schrödinger equation is a powerful tool for description of BEC dynamics, all the studies of BEC in networks used so far tight binding and statistical mechanics-based approaches [17-22]. Moreover, most of the studies of this problem do not discuss experimental realization of BEC in networks. Planar BEC can be experimentally realized in surface optical traps [33], superconductive BEC for exitons in planar systems [34] and atom chip films [35]. All these systems can be constructed in branched form, in which the BEC standing wave can be described by Eq. (1). In this case, parameter $\mu$ in Eq. (1) corresponds to the chemical potential, while $|\psi|^{2}$ describes number of atoms in condensate. The transition from a planar to a one-dimensional BEC dynamics corresponding to the shrinking limit of the above fat graph can then be treated similarly to that in the Ref. [36]. Other types of networks and branched systems where planar and one-dimensional 

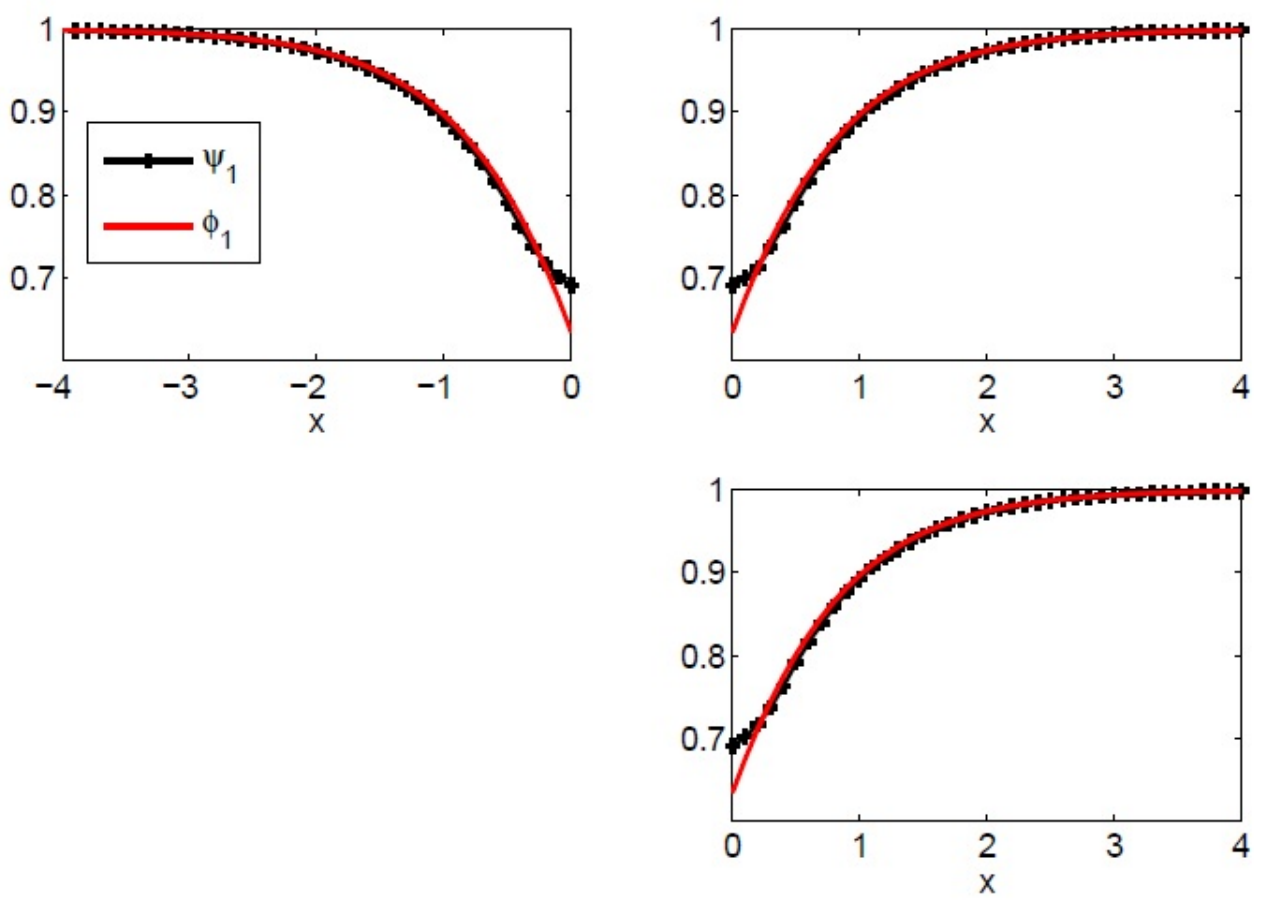

FIG. 4. Dark soliton solution of repulsive NLSE on a fat graph at $\epsilon=0.3, \mu=1, \alpha=2$

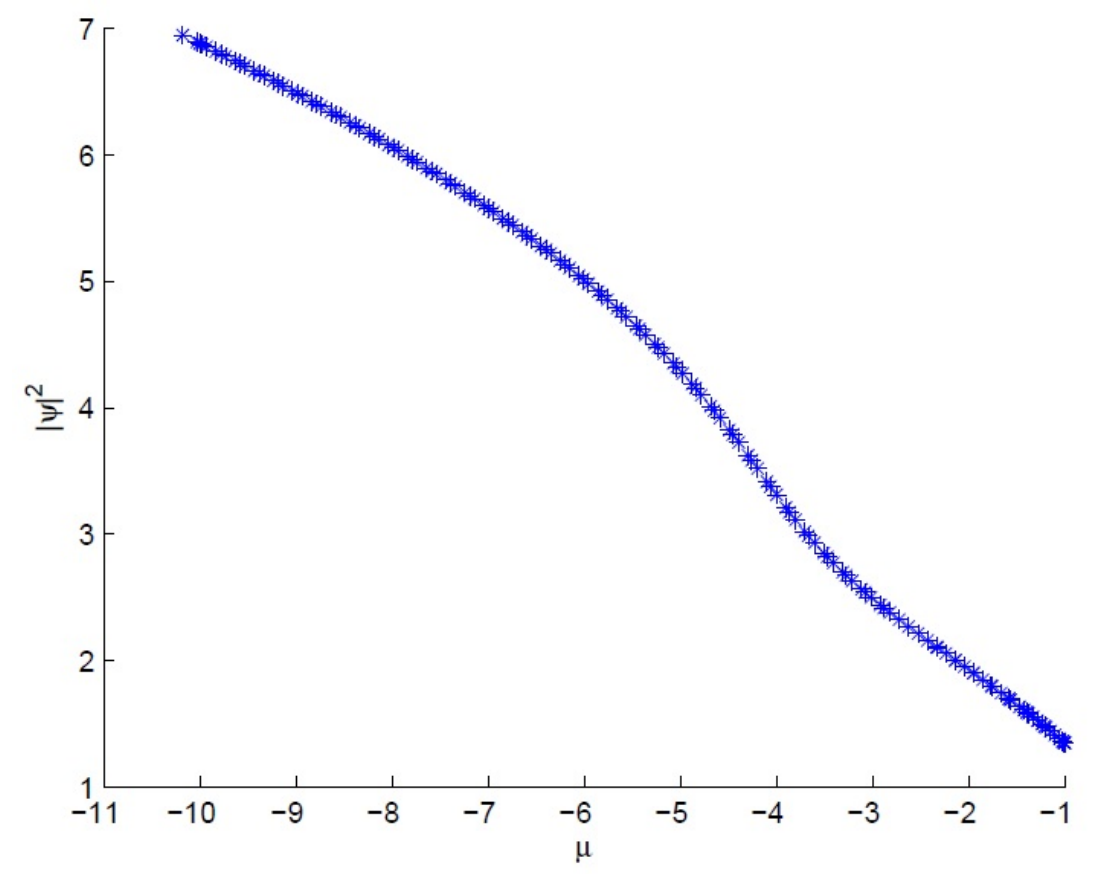

FIG. 5. Chemical potential dependence of the solution of NLSE on a fat graph at the vertex for $\epsilon=0.5, \quad \alpha=0.5$

BEC can occur are the different types of Josephson junctions [35, 37, 38]. Networks or branched structures of Josephson junctions can be realized in different versions [39-43]. Planar Josephson junctions can be fabricated using different techniques $[44,45]$. The standing wave states of the condensate in planar Josephson network can be described by our model. Conducting polymers are also branched systems where BEC can be experimentally realized [22]. 
Networks of planar optical waveguides and fibers. Optical waveguides are systems where the wave propagation can be described by the linear or nonlinear Schrödinger equation. The stationary nonlinear Schrödinger equation describes standing wave modes in such waveguides. Optical waveguides and fibers can be realized in linear, planar and cylindric forms. Few papers discuss different ways for experimental realizations of planar waveguides [46,47]. Networks of such waveguides are of importance from a practical importance viewpoint. In particular, modern optical telecommunication technology requires the use of such waveguide networks rather than separate fibers. Earlier branching of nonlinear optical waves in Y-junction optical media was discussed in the Refs. [23,48]. However, these works did not use the above fat or metric graph approaches. In a branched optical system, the function $\psi$ in Eq. (1) describes amplitude of the wave, while quantity $\mu$ corresponds to the propagation constant. By exploring the shrinking limit of NLSE on a fat graph, one can determine the minimal width of the fibers for which wave transport in such networks can be considered as one dimensional. Also, the above model can be useful for fabrication the materials and devices with tunable optical properties.

Standing waves in DNA. Remarkable branched structures where solitons and nonlinear waves can appear are DNA strands. Depending on the model and approach, such waves can be described by either the sine-Gordon equation or nonlinear Schrödinger equations [49-51]. Within the so-called Peyrard-Bishop model, the nonlinear dynamics of DNA base pairs are described by the NLSE. The base pair of DNA has a branched structure and can be considered as a star graph. Realistic wave motion in DNA is two-dimensional and the transverse component of the oscillations can play important role in DNA dynamics. Therefore, studying the shrinking limit allows one to determine the boundary between the one- and two-dimensional approaches. The two dimensional model of DNA dynamics was considered earlier in [49-51].

Conducting polymers. Polymers are molecular networks having complicated topology whose structural units can be often reduced to a star graph. One type of polymer, which are called conjugated polymers, can exhibit metallic or semiconductor properties. Conducting polymers have attracted much attention due their wide range of electronic applications [24,25]. Charge and spin excitations and their transport in conducting polymers can have solitons and standing nonlinear waves described by NLSE. The motion of such waves is usually two-dimensional but can be effectively described using a one dimensional form. Due to the finite size of its branching points, a polymer chain can be considered as a fat graph. The above NLSE on fat graph and its shrinking limit can be a powerful tool for describing charge and spin carriers dynamics in conducting polymers. This can be an especially effective method for charge transport, recombination and separation in polymer-based materials such as light-emitting diodes, organic solar cells etc.

Capillary networks. Solitons and nonlinear standing waves appear in the hydrodynamics of capillary systems, where fluid dynamics can be described by the NLSE [52]. A planar version of capillary systems as discussed in [52-57]. Nonlinear wave in branched capillary systems should be described by NLSE on a fat graph. The connection point of such network has finite size. Our model allows one to estimate the characteristic width of a capillary tube for which which wave motion in such network could be considered linear.

\section{Conclusions}

In this work, we studied the stationary nonlinear Schrödinger equation on fat graphs by focusing on metric graph transition in the shrinking limit. Analytical estimates for the boundary condition shrinking are obtained. It is shown that in the shrinking limit, fat graph boundary conditions reproduce those for the metric graph. Such a convergence is also shown on the basis of numerical treatment of NLSE on a fat graph. Detailed discussion of the potential application of the model to BEC in networks, branched optical materials, DNA double helix, conducting polymers and capillary networks is discussed. The model can be extended for other fat graph topologies, such as tree, ring and complete graphs. The above study allows one to determine the border between planar and linear motion in branched systems, where particle and wave transport is effectively considered as one dimensional. The results of this paper can be useful for problems of engineering materials and devices based on branched optical and electronic structures.

\section{Acknowledgement}

This work is supported by a grant from the Volkswagen Foundation. We thank Hannes Uecker for his valuable comments on the formulation of the problem and for the help in the numerical part of the work.

\section{References}

[1] Albert R., Barabasi A-L. Statistical mechanics of complex networks. Rev. Mod.Phys. A, 2002, 74, P. 47.

[2] Cohen R., Havlin S. Complex Networks: Structure, Robustness and Function. Cambridge University Press, 2010. 
[3] Sobirov Z., Matrasulov D., Sabirov K., Sawada S., and Nakamura K. Integrable nonlinear Schrödinger equation on simple networks: Connection formula at vertices. Phys. Rev. E, 2010, 81, P. 066602.

[4] Adami R., Cacciapuoti C., Finco D., and Noja D. Fast solitons on star graphs. Rev. Math. Phys., 2011, 23, P. 409.

[5] Adami R., Cacciapuoti C., Finco D., and Noja D. Stationary states of NLS on star graphs. Europhys. Lett., 2012, 100, P. 10003.

[6] Adami R., Cacciapuoti C., Finco D., and Noja D. On the structure of critical energy levels for the cubic focusing NLS on star graphs. $J$. Phys. A: Math. Theor, 2012, 45, P. 192001.

[7] Adami R., Noja D., and Ortoleva C. Orbital and asymptotic stability for standing waves of a NLS equation with concentrated nonlinearity in dimension three. J. Math. Phys., 2013, 54, P. 013501.

[8] Sabirov K.K., Sobirov Z.A., Babajanov D., and Matrasulov D.U. Stationary nonlinear Schrödinger equation on simplest graphs. Phys. Lett. A, 2013, 377, P. 860 .

[9] Noja D. Nonlinear Schrödinger equation on graphs: recent results and open problems. Philos. Trans. R. Soc. A, 2014, 372, P. 20130002.

[10] Cacciapuoti C., Finco D., and Noja D. Topology-induced bifurcations for the nonlinear Schrdinger equation on the tadpole graph. Phys. Rev. E, 2015, 91, P. 013206.

[11] Exner P., Post O. Approximation of quantum graph vertex couplings by scaled Schroedinger operators on thin branched manifolds. $J$. Phys. A: Math. Theor., 2009, 42, P. 415305.

[12] Dell'Antonio G.F. and Costa E. Effective Schrödinger dynamics on $\epsilon$-thin Dirichlet waveguides via quantum graphs: I. Star-shaped graphs. J. Phys. A, Math. Theor., 2010, 43, P. 474014, 23.

[13] Exner P., Post O. A General Approximation of Quantum Graph Vertex Couplings by Scaled Schrdinger Operators on Thin Branched Manifolds. Commun. Math. Phys., 2013, 322, P. 207-227.

[14] Uecker H., Grieser D., Sobirov Z., Babajanov D. and Matrasulov D. Soliton transport in tubular networks: Transmission at vertices in the shrinking limit. Phys. Rev. E, 2015, 91, P. 023209.

[15] Caputo J.-G., Dutykh D. Nonlinear waves in networks: a simple approach using the sine-Gordon equation. Phys. Rev. E, 2014, 90, P. 022912.

[16] Kosugi S. Semilinear Elliptic Equations on Thin Network-Shaped Domains with Variable Thickness. Journal of Differential Equations, 2002, 183, P. 165.

[17] Bianconi G., Barabasi A-L. Bose-Einstein Condensation in Complex Networks. Phys. Rev. Lett., 2001,86 , P. 5632.

[18] Burioni R., Cassi D., Meccoli I., Rasetti M., Regina S., Sodano P. and Vezzani A. Bose-Einstein condensation in inhomogeneous Josephson arrays. Eur. Phys. Lett., 2000, 52, P. 251.

[19] Burioni R., Cassi Rasetti D., Sodano P. and Vezzani A. Bose-Einstein condensation on inhomogeneous complex networks. J. Phys. B, 2001, 34, P. 4697.

[20] Brunelli I., Giusiano G., Mancini F.P., Sodano P. and Trombettoni A. Topology-induced spatial BoseEinstein condensation for bosons on star-shaped optical networks. J. Phys. B, 2004, 37, P. S275.

[21] Burioni R., Cassi D., Sodano P., Trombettoni A. and Vezzani A. Topological filters and high-pass/low-pass devices for solitons in inhomogeneous networks. Phys. Rev. E, 2006, 73, P. 066624.

[22] Castellanos E., Chacon-Acosta G. Polymer BoseEinstein condensates. Phys. Lett. B, 2013, 722, P. 119.

[23] Leo G., Assanto G. Multiple branching of vectorial spatial solitary waves in quadratic media. Opt. Commun., 1998,146, P. 356.

[24] Heeger A.J., Kivelson S., Schrieffer J.R., and Su W.-P. Solitons in conducting polymers. Rev. Mod. Phys. A, 1988, 60, P. 781.

[25] Heeger A.J. Semiconducting and metallic polymers: The fourth generation of polymeric materials. Rev. Mod. Phys. A, 2001, 73, P. 681.

[26] Exner P. and Post O. Convergence of spectra of graph-like thin manifolds. J. Geom. Phys., 2005, 54, P. 77.

[27] Molchanov S. and Vainberg B. Scattering Solutions in Networks of Thin Fibers: Small Diameter Asymptotics. Commun. Math. Phys., 2007, 273, P. 533.

[28] Exner P. and Post O. Convergence of resonances on thin branched quantum wave guides. J. Math. Phys., 2007, 48, P. 092104.

[29] Grieser D., Analysis on graphs and its applications. Isaac Newton Institute, Cambridge, UK, January 8 - June 29, 2007 (AMS, 2008), P. 565-593.

[30] Post O. Spectral analysis on graph-like spaces. Berlin: Springer, 2012.

[31] Jimbo S. Singular perturbation of domains and semilinear elliptic equations. J. Fac. Sci., U. Tokyo, Sect. A. Math., 1988,35 P. 27.

[32] Gilbarg D.,Trudinger N., Elliptic Partial Differential Equations of Second Order. Springer, 1977.

[33] Butov L.V., Lai C.W., Ivanov A.L., Gossard A.C., Chemla D.S. Towards BoseEinstein condensation of excitons in potential traps. Nature, 2002, 417, P. 47.

[34] Ben-Aryeh Y.. Bose-Einstein Condensation of Excitons in Planar Systems and Superconductive Phase Transition Temperature. J. Supercond. and Nov. Magn., 2015, 28, P. 3211.

[35] Gati R. and Oberthaler M.K. Molecular imaging using recolliding electrons. J. Phys. B, 2007, 40, P. R61.

[36] Carr L.D., Leung M.A. and Reinhardt W.P. Dynamics of the Bose-Einstein condensate: quasi-one-dimension and beyond. J. Phys. B, 2000, 33, P. 3983.

[37] Burger F.S.C.S., Fort C., Maddaloni P., Minardi F., Trombettoni A., Smerzi A., and Inguscio M. Josephson junction arrays with BoseEinstien condensates. Science, 2001, 293, P. 843.

[38] Levy S., Lahoud E., Shomroni I., and Steinhauer J. The ac and dc Josephson effects in a Bose-Einstein condensate. Nature, 2007, 449, P. 579.

[39] Hasegawa A., Optical Solitons in Fibers. Springer, 1990.

[40] Hall B.V., Whitlock S., Scharnberg F., Hannaford P. and Sidorov A. A permanent magnetic film atom chip for BoseEinstein condensation. J. Phys. B, 2006, 39, P. 27.

[41] Rychtarik D., Engeser B., Nagerl H.-C., and Grimm R. Two-Dimensional Bose-Einstein Condensate in an Optical Surface Trap. Phys. Rev. Lett., 2004, 92, P. 173003.

[42] De Luca R., Romeo F. Electrodynamic response of current-biased elementary cubic networks of Josephson junctions. Phys. Rev. B, 2002, 66, P. 024509.

[43] Giuliano D. and Sodano P. Pairing of Cooper pairs in a Josephson junction network containing an impurity. Europhys. Lett., 2013, 103, P. 57006 . 
[44] Krasnov V.M., Ericsson O., Intiso S., Delsing P., Oboznov V.A., Prokofiev A.S., Ryazanov V.V. Planar SFS Josephson junctions made by focused ion beam etching. Physica C, 2005, 418, P. 16.

[45] Nussbaum J., Schmidt Th.L., Bruder Ch., and Tiwari R.P. Josephson effect in normal and ferromagnetic topological insulator planar, step and edge junctions. Phys. Rev. B, 2014, 90, P. 045413.

[46] Shandarov, Kip D., Wesner M. and Hukriede J. Observation of dark spatial photovoltaic solitons inplanar waveguides in lithium niobate. J. Opt. Soc. Am. B, 2000, 2, P. 500.

[47] Eisenberg H.S., Morandotti R., Silberberg Y., Arnold J.M., Pennelli G., and Aitchison J.S. Optical discrete solitons in waveguide arrays. I. Soliton formation. J. Opt. Soc. Am. B, 2002, 19, P. 2938.

[48] Santos M.C. Dynamic Y-branched structures in quadratic nonlinear media. Opt. Commun., 2000, 180 , P. 167.

[49] Yakushevich L.V., Nonlinear Physics of DNA. Wiley, Chichester, 2004.

[50] Peyrard M. Nonlinear dynamics and statistical physics of DNA. Nonlinearity, 2004, 17, P. R1.

[51] Zdravkovica S., Sataric M.V. Nonlinear Schrdinger equation and DNA dynamics. Phys. Lett. A, 2008, 373, P. $126-132$.

[52] Rasmussen J. and Stiassnie M. Multiple gravity-capillary wave-forms near the minimum phase-speed. J. Fluid. Mech., 1999, 384, P. 93.

[53] Sobirov Z.A., Akhmedov M.I., Karpova O.V., Jabbarova B. Linearized KdV equation on a metric graph. Nanosystems: Physics, Chemistry, Mathematics, 2015, 6(6), P. 757-761.

[54] Vidal E.J.G.G., Lima R.P.A., and Lyra M.L. Bose-Einstein condensation in the infinitely ramified star and wheel graphs. Phys. Rev. E, 2011, 83, P. 061137.

[55] de Oliveira I.N., dos Santos T.B., de Moura F.A.B.F., Lyra M.L., and Serva M. Critical behavior of the ideal-gas Bose-Einstein condensation in the Apollonian network. Phys. Rev. E, 2013, 88, P. 022139.

[56] Dalfovo F., Giorgini S., Pitaevskii L.P., and Stringari S. Theory of Bose-Einstein condensation in trapped gases. Rev. Mod. Phys. A, 1999, 71, P. 463.

[57] Muto V., Lomdahl P.S., and Christiansen P.L. Two-dimensional discrete model for DNA dynamics: Longitudinal wave propagation and denaturation. Phys. Rev. A, 1990, 42, P. 7452. 